\title{
An Approximation Approach to Non-Gaussian Filtering with Accuracy Analysis
}

\author{
Mehdi Nouri-Shirazi*, Nobuo Sannomiya* and Yoshikazu Nishikawa*
}

\begin{abstract}
We consider a discrete linear dynamical system with known Gaussian system disturbances and known non-Gaussian observation noises. We propose an approximation filter which is optimal in the sense that the trace of the estimation error-covariance matrix is minimum for the optimal score function belonging to a preassigned class of score functions. Then, a recursive system of equations is derived for a scalar dynamical system which gives a lower and an upper bound of the filter's performance. In order to illustrate the properties of the proposed filter, a scalar system is considered. Results of simulation are presented for two cases; a) mixture Gaussian observation noise, and b) Cauchy observation noise. The lower and upper bounds of the filter's performance are also included for the case $b$.
\end{abstract}

Key Words: Kalman filter, non-Gaussian filtering, non-linear filtering, elliptical process

\section{INTRODUCTION}

The linear optimal filtering theorems and algorithms are clean and powerful. They are used widely even if there is an obvious deviation from the optimal conditions. The fact is that the performance calculations and the filter gain calculations can be performed off-line; and the equations involved in on-line calculations are no more complicated than the system dynamics.

In practical applications it is well known that the dogma of normality should be abandoned ${ }^{1}$. It is a fact that the linear optimal filter (Kalman filter) might be sensitive to the deviation from normality. It has been a motivation for considering filters which can perform fairly well in non-

* Faculty of Engineering, Kyoto University, Sakyoku, Kyoto

(Received July 26, 1984)

(Revised October 8, 1984)
Gaussian environments while preserving, in some degree, the merits of the linear optimal filters.

Sorenson and Alspach" ${ }^{2}$ introduced the Gaussian sums approach. In their method, the a posteriori density function is approximated by a sum of Gaussian density functions, and to each Gaussian density function an optimal filter is assigned. Although the method is conceptually promising, it is not attractive in application because of its complexity. That is to say, the filter so constructed is not implementable because the number of terms in the Gaussian sums increases exponentially in the course of the filter's activity.

Masreliez ${ }^{3)}$ proposed an approximation nonGaussian filter. His method is based on the assumption that the density function of the estimation error is Gaussian. By this assumption he could stop the number of the terms in the Gaussian sums approximation to increase exponentially as the filter propagates. Consequently, his filter achieves a remarkable saving in computation time. It might be considered as an excellent characteristic of his approach. However, there are several drawbacks with this approach. One is that the score function of the filter can not be expressed analytically. Dealing with this problem, Tsai and Kurz" introduced a method called $m$ interval polynomial approximation (MIPA). The essence of their method is to divide the score function into $m$ intervals and then to interpolate the $m$ points with linear functions. The effectiveness of the method is questionable, because it is not clear how to select these $m$ points. For example, the performance of the filter is very sensitive to the shape of the score function at the interval of low value residuals.

The other problem with the Masreliez's filter 
is that the shape of the score function is too complex for analysis of the filter performance, through the available methods, in advance of the filter implementation. Hence, Masreliez's filter is still far from being a suitable competitor of the Kalman filter in the realm of the practice.

In this paper we propose another approach for the construction of an approximation filter. In this approach, a class of score functions having a preassigned structure with a finite number of free parameters is considered. Then, the filter is constructed by minimizing the trace of the errorcovariance matrix with respect to these parameters. Furthermore, using a geometrical method known as the convex hull method developed by Levin ${ }^{5)}$, we give a recursive system of equations for the lower and upper bounds accuracy of the proposed filter. Simulation results show that, in the expense of a little degradation in filter's performance, we can construct an approximation filter having verv simple structure comparable, with the linear optimal filter.

\section{Problem Statement}

A linear discrete-time system is described by the following state-space equations

$$
\begin{aligned}
& x_{k}=F_{k-1} x_{k-1}+w_{k-1} \\
& y_{k}=H_{k} x_{k}+v_{k} \quad k=1,2, \cdots
\end{aligned}
$$

$x_{k}$ is the $n \times 1$ state vector and $y_{k}$ is the $r \times 1$ observation vector. $w_{k}$ and $v_{k}$ are disturbance vectors of appropriate dimensions. Taking the minimum variance estimator which is given by the conditional expectation $\hat{x}_{k}=E\left[x_{k} \mid Y^{k}\right]$, where $Y^{k} \triangleq$ $\left(y_{1}, y_{2}, \cdots, y_{k}\right)$, we want to estimate the state vector $x_{k}$.

We do this under the following assumptions: $\left\{w_{k}\right\}$ and $\left\{v_{k}\right\}$ are mutually independent white processes. $w_{k}$ is Gaussian with the zero mean and the known covariance matrix $Q_{k} . v_{k}$ is known symmetrical heavy-tailed non-Gaussian. The initial state vector $x_{0}$ is Gaussian with the mean $\bar{x}_{0}$ and the covariance matrix $P_{0}$ which is independent of $w_{k}$ and $v_{k}$ for all $k$.

\section{Masreliez's Approximation Filter}

Masreliez $z^{3}$ assumed that the conditional state prediction density $p\left(x_{k} \mid Y^{k-1}\right)$ is Gaussian. Then, he derived his filter as a linear transformation of the score function vector. The conditional expectation $\hat{x}_{k}$ and its conditional error covariance $P_{k} \triangleq E\left[\left(\hat{x}_{k}-x_{k}\right)\left(\hat{x}_{k}-x_{k}\right)^{T} \mid Y^{k}\right]^{\prime}$ are given as follows :

Time update equations;

$$
\begin{aligned}
& \bar{x}_{k}=F_{k-1} \hat{x}_{k-1} \\
& M_{k}=F_{k-1} P_{k-1} F_{k-1}^{T}+Q_{k-1}
\end{aligned}
$$

Measurement update equations ;

$$
\begin{aligned}
& \hat{x}_{k}=\bar{x}_{k}+M_{k} H_{k}^{T} g_{k}\left(z_{k}\right) \\
& P_{k}=M_{k}-M_{k} H_{k}^{T} G_{k}\left(z_{k}\right) H_{k} M_{k}
\end{aligned}
$$

where $z_{k}$ is the residual vector, i. e., $z_{k}=y_{k}-H_{k} \bar{x}_{k}$. The score function vector $g_{k}\left(z_{k}\right)$ is an $r \times 1$ vector with the $i$-th component

$$
g_{k}^{i}\left(z_{k}\right)=-\left[\partial p\left(z_{k} \mid Z^{k-1}\right) / \partial z_{k}^{i}\right] p^{-1}\left(z_{k} \mid Z^{k-1}\right)
$$

where $Z^{k-1} \triangleq\left(z_{1}, z_{2}, \cdots, z_{k-1}\right)$ and $p\left(z_{k} \mid Z^{k-1}\right)$ is the conditional observation prediction density. $G_{k}\left(z_{k}\right)$ is an $r \times r$ matrix with the $i j$-th element

$$
G_{k}^{i j}\left(z_{k}\right)=\partial g_{k}^{i}\left(z_{k}\right) / \partial z_{k}^{j}
$$

\section{Comments :}

a) Except for a simple case where the $v_{k}$ is a Gaussian mixture, the convolution needed for generating $p\left(z_{k} \mid Z^{k-1}\right)$ is considerably involved. It stops the filter to be attractive for on-line use. A remedy is to investigate the shape of the score function established by the off-line calculation, and then to introduce an approximation to the function suitable for on-line data processing. By so doing, the on-board computer memory is occupied partly with parameters defining the score functions. Tsai and $\mathrm{Kur}^{4}{ }^{4}$ proposed MIPA Kalman filters where all parameters representative of the score functions are calculated adaptively. Nevertheless, it increases an on-line computational burden, especially when the number of intervals is large.

b). One of the advantages of the Kalman filter consists in the fact that the conditional errorcovariance $P_{k}$ is not a function of the observation data. That is, the conditional error-covariance $P_{k}$ and the unconditional error-covariance,

$$
P_{k}^{*}=E\left[P_{k}\right] \text {, }
$$

are equal. The expectation is taken with respect to $p\left(z_{k} \mid Z^{k-1}\right)$. It means that in the design level, an exact knowledge about the accuracy of the linear optimal filter is available a priori. On the 
contrary, $P_{k}$ is a function of the observation data in non-Gaussian filtering. It is possible (at least theoretically) to estimate the filtering performance by averaging $P_{k}$, though it is not a simple task. In the case of the Masreliez approximation filter, the situation is more unfavourable. Error-covariance $P_{k}^{*}$ is derived from (6) based on the assumption that $p\left(x_{k} \mid Z^{k-1}\right)$ is Gaussian. There is no idea about the sensitivity of $P_{k}^{*}$ to the violation of this assumption. It might be claimed that, if the system disturbance is Gaussian, $p\left(x_{k} \mid Z^{k-1}\right)$ is almost a Gaussian density, a claim which can not be justified theoretically.

\section{Proposed Approximation Filter}

We consider an $r$-dimensional Gaussian mixture family, and an $r$-dimensional elliptical family defined, respectively, by

$$
\begin{aligned}
\mathscr{F}_{G}^{r}= & \left\{p(\cdot) \mid p(\cdot)=a_{1} \Phi_{R_{1}}(\cdot)+a_{2} \Phi_{R_{2}}(\cdot),\right. \\
& \left.a_{1}+a_{2}=1\right\} \\
\mathscr{F}_{E}^{r}= & \{p(\cdot) \mid p(\cdot)=s(\cdot \mid f, R)\}
\end{aligned}
$$

where $\Phi_{R}$ is the $r$-dimensional Gaussian density with the zero mean and the covariance matrix $R$. In (10), the elliptical density function $s(\cdot \mid f, R)$ is defined by

$$
s(\cdot \mid f, R) \triangleq \int_{0}^{\infty} f(t) \Phi_{t^{-1} R}(\cdot) d t
$$

where $R$ is the characteristic matrix and $f(t)$ is a scalar function defined on $0<t<\infty$.

Several practically interesting heavy-tailed nonGaussian densities such as Laplace, Cauchy, and student densities belong to the elliptical family ${ }^{6}$.

The following lemma is due to Masreliez and $\operatorname{Martin}^{7)}$

Lemma : Let $s=s_{1}+s_{2}$ with $s_{1}$ and $s_{2}$ independent raridom variables, and $p\left(s_{1}\right)=\Phi_{R_{0}}\left(s_{1}\right)$. If $p\left(s_{2}\right) \in \mathcal{F}_{G}^{r}$ or $p\left(s_{2}\right) \in \mathscr{F}_{E}^{r}$, then there exists an $r \times r$ transformation matrix $T$ such that the density of $z=T s$ satisfies the following two properties,

$$
p 1: p\left(z^{1}, z^{2}, \cdots, z^{i}, \cdots, z^{r}\right)=p\left(z^{1}, z^{2}, \cdots,-z^{i}, \cdots, z^{r}\right),
$$
for each $i$.

p2: All marginal p.d.f. of $p$ are members of $\mathscr{F}_{G}^{1}$ when $p\left(s_{2}\right) \in \mathscr{F}_{G}^{r}$, or of $\mathscr{F}_{E}^{1}$ when $p\left(s_{2}\right) \in \mathcal{F}_{E}^{r}$.

Let $p\left(x_{k} \mid Y^{k-1}\right)$ be Gaussian with the mean $\bar{x}_{k}$, and the covariance $M_{k}$. As it will become clear later, $M_{k}$ is in fact the unconditional a priori covariance. Let $T_{k}$ be such that the residual

$$
z_{k}=T_{k} y_{k}-T_{k} H_{k} \bar{x}_{k}=T_{k}\left[H_{k}\left(x_{k}-\bar{x}_{k}\right)+v_{k}\right](12)
$$

satisfies properties $p 1$ and $p 2$. Then, we hypothesize the following concrete structure for our filter

$$
\hat{x}_{k}=\bar{x}_{k}+H_{k}^{T} T_{k}^{T} A_{k} \Psi_{k}\left(z_{k}\right)
$$

where $A_{k}$ is an $r \times r$ diagonal matrix with the diagonal elements $a_{k}^{i}$ to be chosen appropriately. The score function vector $\Psi_{k}\left(z_{k}\right)$ has elements $\Psi_{k}^{i}\left(z_{k}\right)=\psi_{k}^{i}\left(z_{k}^{i}\right)$ where

$$
\psi_{k}^{i}\left(z_{k}^{i}\right) \triangleq \begin{cases}-1 & \text { for } z_{k}^{i} \leqq-b_{k}^{i} \\ z_{k}^{i} / b_{k}^{i} & \text { for }\left|z_{k}^{i}\right|<b_{k}^{i} \\ +1 & \text { for } z_{k}^{i} \geqq b_{k}^{i}\end{cases}
$$

$b_{k} \triangleq\left[b_{k}^{1}, \cdots, b_{k}^{r}\right]^{T}$ is a vector left to be chosen. With this filter, the unconditional error-covariance is given by

$$
\begin{aligned}
V_{k}\left(A_{k}, b_{k}\right)= & M_{k}-H_{k}^{T} T_{k}^{T} A_{k} \Omega_{k} T_{k} H_{k} M_{k} \\
& -M_{k} H_{k}^{T} T_{k}^{T} \Omega_{k} A_{k} T_{k} H_{k} \\
& +H_{k}^{T} T_{k}^{T} A_{k} \Lambda_{k} A_{k} T_{k} H_{k}
\end{aligned}
$$

where $\Omega_{k}$ and $\Lambda_{k}$ are diagonal matrices with diagonal elements $\Omega_{k}^{i i}=E\left[\psi_{k}^{i}\left(z_{k}^{i}\right)^{\prime}\right]$ and $\Lambda_{k}^{i i}=E$ $\left[\left(\phi_{k}^{i}\left(z_{k}^{i}\right)\right)^{2}\right]$, respectively. A prime denotes differentiation with respect to the argument $z_{k}^{i}$. For proof, see Appendix A.

The values of $A_{k}$ and $b_{k}$ might be chosen by solving the following deterministic optimization problem :

$$
\min \operatorname{tr}\left[V_{k}\left(A_{k}, b_{k}\right)\right]
$$

Since $A_{k}, \Omega_{k}$ and $\Lambda_{k}$ are diagonal matrices, minimization of (16) is decomposed into $r$ independent minimization subproblems, each having only two parameters $a_{k}^{i}$ and $b_{k}^{i}$. Furthermore, the function to be minimized is quadratic with respect to $a_{k}^{i}$. Consequently, the solution of (16) can be obtained by solving $r$ one-dimensional searches for $b_{k}^{i}: i=1,2, \cdots, r$.

If we denote the optimal values of $A_{k}$ and $b_{k}$ by $A_{k}^{*}$ and $b_{k}^{*}$, respectively, the unconditional error-covariance is obtained as

$$
P_{k}^{*}=V_{k}\left(A_{k}^{*}, b_{k}^{*}\right)
$$

and the filter's equations are given as

measurement update equations ;

$$
\hat{x}_{k}=\bar{x}_{k}+H_{k}^{T} T_{k}^{T} A_{k}^{* T} \Psi_{k}^{*}\left(z_{k}\right)
$$

time update equations;

$$
\begin{aligned}
& \bar{x}_{k}=F_{k-1} \hat{x}_{k-1} \\
& M_{k}=F_{k-1} P_{k-1}^{*} F_{k-1}^{T}+Q_{k-1} .
\end{aligned}
$$

In (18), $\Psi_{k}^{*}$ has the $i$-th element $\phi_{k}^{i *}\left(z_{k}^{i}\right)$, where it 
M. Nouri-Shirazi, N. SANNOMiYA and Y. Nishikawa :

An Approximation Approach to Non-Gaussian Filtering with Accuracy Analysis

is given by (14) with $b_{k}^{i}=b_{k}^{i *}$. From (15) and (17), it is obvious that $P_{k-1}^{*}$ is the uncoditional error-covaiance. Hence, $M_{k}$ in (20) is unconditional as well.

\section{Accuracy Analysis of the Proposed Approximation Filter}

Levin $^{5)}$ proposed a geometrical approach, socalled the method of convex hulls, to the analysis of the accuracy of a nonlinear filter having a bounded score function. For definiteness he considered a one-step ahead predictor having the score function

$$
\phi(z)= \begin{cases}K z & \text { for }|z| \leqq b \\ 0 \text { for }|z|>b\end{cases}
$$

Then he derived a system of recursive equations which gives nontrivial lower and upper bounds of the mean-square error of the predictor.

Here, we adopt his approach and construct a system of recursive equations for our approximation filter in the case where $n=1$ and $r=1$.

\subsection{The method of convex hulls}

Let us consider two functions $f_{1}(e)$ and $f_{2}(e)$, $e \in R^{1}$. Let e be a random variable with unknown density function having a bounded expectation

$$
E\left[f_{1}(e)\right] \leqq a<\infty
$$

We want to give an optimal estimate of $E\left[f_{2}(e)\right]$ under the condition (21). For this purpose, let us consider the following parametric curve defined on $R^{2}$ :

$$
C=\left\{(x, y) \mid x=f_{1}(e), y=f_{2}(e) ; e \in R^{1}\right\}
$$

If the density of $e, p(e)$, scans all possible densities in the space of density functions, then the points with coordinates

$$
\begin{aligned}
& x(p)=E\left[f_{1}(e)\right]=\int f_{1}(e) p(e) d e \\
& y(p)=E\left[f_{2}(e)\right]=\int f_{2}(e) p(e) d e
\end{aligned}
$$

form the convex hull of the curve $C, \mathrm{Co}(C)$.

Now we introduce the following four functions defined on the projection of $\mathrm{Co}(C)$ on the $x$ axis:

$$
\begin{aligned}
& L_{i i}(a)=\min \quad \text { inf } y \\
& x \geqq a \quad\{y \mid(x, y) \in \mathrm{Co}(C)\} \\
& L_{d}(a)=\min \quad \text { inf } y \\
& x \leqq a \quad\{y \mid(x, y) \in \operatorname{Co}(C)\} \\
& U_{i}(a)=\max \quad \sup y \\
& x \leqq a \quad\{y \mid(x, y) \in \mathrm{Co}(C)\}
\end{aligned}
$$

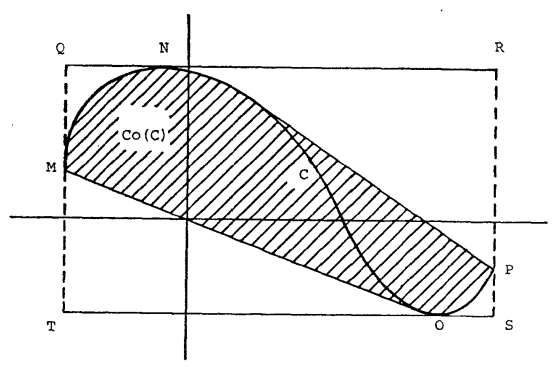

Fig. 1 Lower and upper bounds associated with the curve $C$

$$
\begin{array}{rc}
U_{d}(a)=\max & \sup y \\
x \geqq a & \{y \mid(x, y) \in \operatorname{Co}(C)\}
\end{array}
$$

Figure 1 shows these functions. In this figure, the solid line MNOP represents the parametric curve $C$, and the shaded area is $\operatorname{Co}(C)$. The functions $L_{i}, L_{d}, U_{i}$ and $U_{d}$ are shown by the curves TOP, MOS, MNR and QNP, respectively.

Under the condition (21), the lower and upper bounds of the estimate $E\left[f_{2}(e)\right]$ are given by

$$
L_{d}(a) \leqq E\left[f_{2}(e)\right] \leqq U_{i}(a)
$$

In the same manner, under the condition

$$
\mathrm{E}\left[f_{1}(e)\right] \geqq a
$$

the lower and upper bounds of $E\left[f_{2}(e)\right]$ are

$$
L_{i}(a) \leqq E\left[f_{2}(e)\right] \leqq U_{d}(a)
$$

\subsection{Lower and upper bounds recursive equations}

For simplicity we consider the following scalar system :

$$
\begin{aligned}
& x_{k}=F x_{k-1}+w_{k-1} \\
& y_{k}=H x_{k}+v_{k}
\end{aligned}
$$

with the relevant approximation filter

$$
\hat{x}_{k}=F \hat{x}_{k-1}+\phi_{k}\left(y_{k}-F H \hat{x}_{k-1}\right)
$$

where

$$
\phi_{k}\left(z_{k}\right)= \begin{cases}-K_{k} b_{k} & \text { for } z_{k} \leqq-b_{k} \\ K_{k} z_{k} & \text { for }\left|z_{k}\right|<b_{k} \\ +K_{k} b_{k} & \text { for } z_{k} \geqq b_{k}\end{cases}
$$

The parameters $K_{k}$ and $b_{k}$ are assumed known for all $k$. From (32), (33) and (34), a recursive equation for the estimation error, $e_{k} \triangleq x_{k}-\hat{x}_{k}$, is derived as

$$
e_{k}=F e_{k-1}-\phi_{k}\left(F H e_{k-1}+H w_{k-1}+v_{k}\right)
$$

Now we define the following binary variables:

$$
\begin{aligned}
& I_{k}^{1} \triangleq\left\{\begin{array}{l}
1 \text { for }\left|F H e_{k-1}+H w_{k-1}+v_{k}\right| \leqq b_{k} \\
0 \text { otherwise }
\end{array}\right. \\
& I_{k}^{2} \triangleq 1-I_{k}^{1}
\end{aligned}
$$




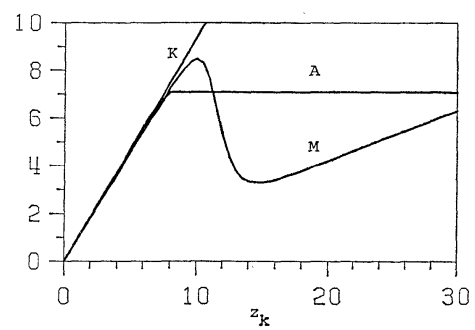

(a)

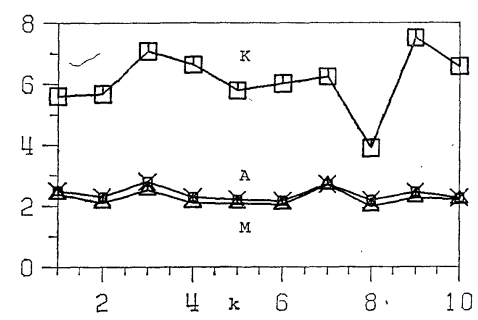

Fig. 2 Comparison among Kalman filter (K), Masreliez's filter (M), and the proposed approximation filter (A) for the case of a mixture Gaussian observation noise,

a) Score function $(k=4)$

b) Experimental error-variance

$$
\begin{aligned}
& I_{k}^{3} \triangleq\left\{\begin{array}{l}
1 \text { for } F H e_{k-1}+H w_{k-1}+v_{k}>b_{k} \\
0 \text { otherwise }
\end{array}\right. \\
& I_{k}^{4} \triangleq \begin{cases}1 \text { for } F H e_{k-1}+H w_{k-1}+v_{k}<-b_{k} \\
0 \text { otherwise }\end{cases}
\end{aligned}
$$

We also define the following parametric curves in the $x y$ plane:

$$
\begin{aligned}
& C_{k}^{1} \triangleq\left\{(x, y) \mid x=e_{k-1}^{2}, y=E\left[e_{k}^{2} \mid e_{k-1}\right]\right\} \\
& C_{k}^{2} \triangleq\left\{(x, y) \mid x=e_{k-1}^{2}, y=\xi_{k}\right\} \\
& C_{k}^{3} \triangleq\left\{(x, y) \mid x=e_{k-1}^{2}, y=\eta_{k}\right\} \\
& C_{k}^{4} \triangleq\left\{(x, y) \mid x=e_{k-1}^{2}, y=E\left[e_{k-1} w_{k-1} I_{k}^{2} \mid e_{k-1}\right]\right\} \\
& C_{k}^{5} \triangleq\left\{(x, y) \mid x=e_{k-1}^{2}, y=E\left[w_{k-1}\left(I_{k}^{3}-I_{k}^{4}\right) \mid e_{k-1}\right]\right\} \\
& C_{k}^{6} \triangleq\left\{(x, y) \mid x=e_{k-1}^{2}, y=E\left[w_{k-1}^{2} I_{k}^{1} \mid e_{k-1}\right]\right\} \\
& C_{k}^{7} \triangleq\left\{(x, y) \mid x=e_{k-1}^{2}, y=E\left[I_{k}^{2} \mid e_{k-1}\right]\right\} \\
& C_{k}^{8} \triangleq\left\{(x, y) \mid x=J_{k}\left(e_{k-1}\right), y=E\left[e_{k}^{2}\left(I_{k+1}^{2}-I_{k}^{2}\right) \mid e_{k-1}\right]\right\} \\
& C_{k}^{9} \triangleq\left\{(x, y) \mid x=J_{k}\left(e_{k-1}\right), y=\zeta_{k}\right\} \\
& C_{k}^{10} \triangleq\left\{(x, y) \mid x=J_{k}\left(e_{k-1}\right), y=E\left[J_{k+1}\left(e_{k}\right) \mid e_{k-1}\right]\right\}
\end{aligned}
$$

where

$$
\begin{aligned}
& \xi_{k} \triangleq E\left[\left(F e_{k-1}-K_{k}\left(F H e_{k-1}+v_{k}\right)\right)^{2} I_{k}^{1} \mid e_{k-1}\right] \\
& \eta_{k} \triangleq E\left[\left(F e_{k-1}-K_{k}\left(F H e_{k-1}+v_{k}\right)\right) w_{k-1} I_{k}^{1} \mid e_{k-1}\right] \\
& \zeta_{k} \triangleq E\left[e_{k}\left(\left(I_{k+1}^{3}-I_{k+1}^{4}\right)-\left(I_{k}^{3}-I_{k}^{4}\right)\right) \mid e_{k-1}\right] \\
& J_{k}(e) \triangleq\left\{\begin{array}{l}
e^{2} \text { for }|e| \leqq b_{k} \\
d_{k}^{2} \text { for }|e|>b_{k}
\end{array}\right.
\end{aligned}
$$

$d_{k}$ is a known constant less than $b_{k}$.

Then the following theorem is established.

Theorem 1: Suppose that $e_{k}$ is given by (36) and
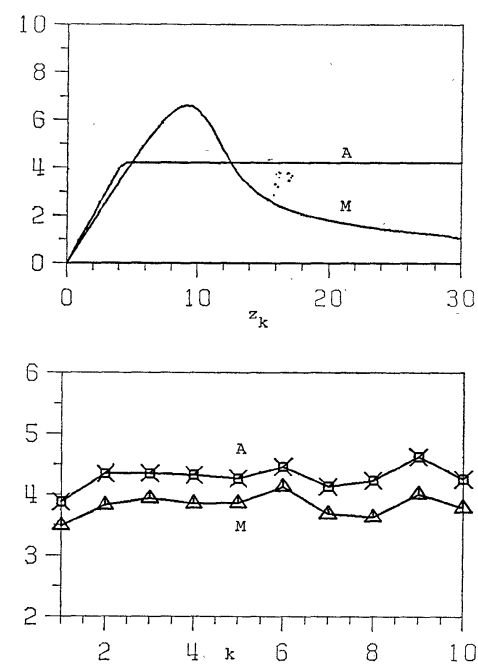

Fig. 3 Comparison between Masreliez's filter $(\mathrm{M})$ and the proposed approximation filter (A) for the case of Cauchy observation noise,
a) Score function $(k=4)$
b) Experimental error-variance

$\rho_{k-1}^{5} \leqq E\left[e_{k-1}^{2}\right] \leqq \rho_{k-1}^{1}$

then the lower and upper bounds of $E\left[e_{k}^{2}\right]$, denoted by $\rho_{k}^{*} \rho_{k}^{5}$ and $\rho_{k}^{1}$, respectively, are determined by the following recursive system of equations:

$$
\begin{aligned}
\rho_{k}^{1}= & U_{i}^{2}\left(\rho_{k-1}^{1}\right)+2 K_{k} H U_{i}^{3}\left(\rho_{k-1}^{1}\right)-2 L_{d}^{3}\left(\rho_{k-1}^{1}\right)+F^{2} \rho_{k-1}^{2} \\
& -2 F K_{k} b_{k} \rho_{k-1}^{3}+K_{k}^{2} b_{k}^{2} U_{i}^{7}\left(\rho_{k-1}^{1}\right)+2 F U_{k}^{4}\left(\rho_{k-1}^{1}\right) \\
& -2 K_{k} b_{k} L_{d}^{5}\left(\rho_{k-1}^{1}\right)+K_{k}^{2} H^{2} U_{i}^{6}\left(\rho_{k-1}^{1}\right) \\
& -2 K_{k} H L_{d}^{6}\left(\rho_{k-1}^{1}\right)+E\left[w_{k-1}^{2}\right] \\
\rho_{k}^{2}= & F^{2} \rho_{k-1}^{2}-2 F K_{k} b_{k} \rho_{k-1}^{3}+K_{k}^{2} b_{k}^{2} U_{i}^{7}\left(\rho_{k-1}^{1}\right) \\
& +2 F U_{i}^{4}\left(\rho_{k-1}^{1}\right)-2 K_{k} b_{k} L_{d}^{5}\left(\rho_{k-1}^{1}\right)-L_{d}^{6}\left(\rho_{k-1}^{1}\right) \\
& +U_{d}^{8}\left(\rho_{k-1}^{4}\right)+E\left[w_{k-1}^{2}\right] \\
\rho_{k}^{3}= & F \rho_{k-1}^{3}-K_{k} b_{k} U_{i}^{7}\left(\rho_{k-1}^{1}\right)+L_{d}^{5}\left(\rho_{k-1}^{1}\right) \\
& +L_{i}^{9}\left(\rho_{k-1}^{4}\right) \\
\rho_{k}^{4}= & L_{i}^{10}\left(\rho_{k-1}^{4}\right) \\
\rho_{k}^{5}= & L_{i}^{1}\left(\rho_{k-1}^{5}\right)
\end{aligned}
$$

with $p\left(e_{0}\right)=p\left(x_{0}\right)$. In the above expressions, the functions $L_{i}^{j}, L_{d}^{j}, U_{i}^{j}$ and $U_{d}^{j}$ are determined from (25) $-(28)$ in which the superscript $j$ corresponds to the number of the curve $C_{k}^{j}$.

For the proof see Appendix B.

\section{Simulation Results}

For investigating the basic features of the proposed filter, a one dimensional system (32)-(33) is considered where $F=0.5, H=1.0$, and $w_{k}$ is 
M. Nouri-Shirazi, N. SANNomiya and Y. Nishikawa :
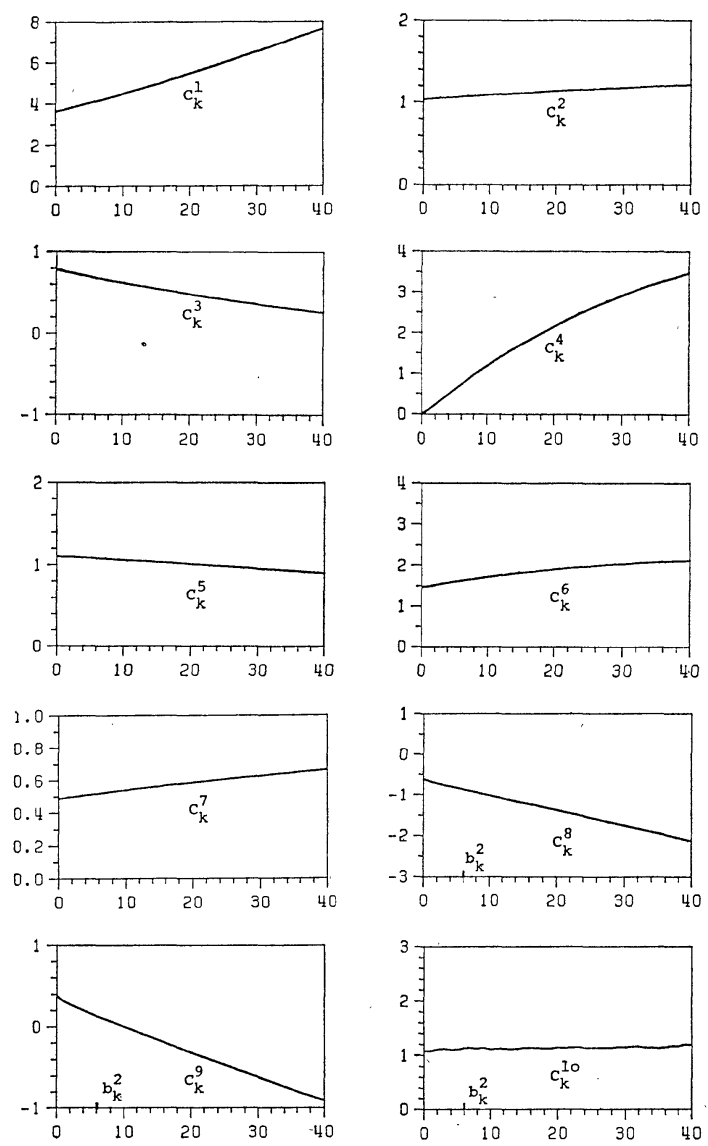

Fig. 4 The graph of the parametric curves defined for estimating the lower and upper bounds of the error-variance $(k=4)$. The horizontal axes show $e_{k-1}^{2}\left(b_{k}^{2}=5.94\right)$

Gaussian with zero mean and constant variace, $Q_{k}=7.3$, for all $k$. Monte-Carlo simulation is performed for two cases; a) $v_{k}$ is a mixture Gaussian

$p\left(v_{k}\right)=0.9 \Phi_{1}\left(v_{k}\right)+0.1 \Phi_{64}\left(v_{k}\right)$ for all $k$, and b) $v_{k}$ is Cauchy

$p\left(v_{k}\right)=1 /\left(\pi\left(1+v_{k}^{2}\right)\right)$ for all $k$,

Several comparisons are made among the Kalman filter, the Masreliez's filter and the proposed filter for the case a, and also between the Masreliez's filter and the proposed filter for the case b. Fig. 2(a) and Fig. 3(a) display the positive part of the relevant filter's score functions, while Fig. 2(b) and Fig. 3(b) show their experimental error-variances. Furthermore, for the case $b$ lower and upper bounds for the proposed filter's error-variance are obtained, based

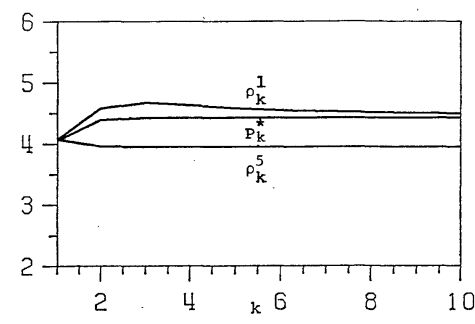

Fig. 5 Theoretical error-variance and the associated lower and upper bounds

on the recursive equations (42)-(46). Fig. 4 shows the parametric curves defined for this purpose.

Fig. 5 represents the lower and upper bounds in addition to the theoretical error-variace (17).

\section{Conclusion}

An approximation filter with very simple structure has been proposed, and its basic properties have been investigated through Monte-Carlo simulation for two cases. A simulation has been carried out for a mixture. Gaussian observation noise as the first case. The performance of the proposed filter shows only a little degradation in spite of a very simple structure, as compared with the Masreliez's filter. The results obtained in this case might induce the belief that there is a trivial relation between the present approach and the MIPA approach. To remove any possible contemplation of this kind, an observation noise with Cauchy density function has been chosen as the second case. The difference between the slopes of the score functions of the Masreliez's filter and the proposed filter for the low value residuals proves the nontrivial relation.

Accuracy analysis has shown that the theoretical error-variance $P_{k}^{*}$ lies between the lower and upper bounds of the error-variance. This fact can be interpreted as follows. In one hand, the normality assumption for the estimation error is valid. On the other hand, the estimation errorvariance carried out off-line gives a reliable information about the quality of the estimation.

Considering the simplicity of the structure, and availability of the information about the quality of the estimation before any implementation, we believe that the proposed approximation filter is 
a suitable competitor of the Kalman filter in non-Gaussian environments.

\section{References}

1) P. J. Huber: The 1972 Wald Lecture Robust Statistics: A Review, Annals of Math. Stat., 43-4, 1041/1067 (1972)

2) H.W. Sorenson and D. L. Alspach: Recursive Bayesian Estimation Using Gaussian Sums, Automatica, 7-4, 465/479 (1971)

3) C. J. Masreliez: Approximate Non-Gaussian Filtering with Linear State and Observation Relations, IEEE Trans. on Automatic Control, AC-20-1, 107/ 111 (1975)

4) C. Tsai and L. Kurz: An Adaptive Robustizing Approach to Kalman Filtering, Automatica, 19-3, 279/288 (1983)

5) I.K. Levin: Accuracy Analysis of a Robust Filter of a Certain Type by the Method of Convex Hulls, Auto. Rem. Control, 41-5, 660/669 (1981)

6) K.C. Chu: Estimation and Decision for Linear Systems with Elliptical Random Processes, IEEE Trans. on Automatic Control, AC-18-5, 499/505 (1973)

7) C. J. Masreliez and R. D. Martin: Robust Bayesian Estimation for the Linear Model and Robustifying the Kalman Filter, IEEE. Trans. on Automatic Control, AC-22-3, 361/371 (1977)

\section{Appendix}

\section{Appendix A : the proof of Equation (15)}

Let us denote

$$
\begin{aligned}
& z_{k}=T_{k} y_{k}-T_{k} H_{k} \bar{x}_{k} \\
& \tilde{x}_{k}=x_{k}-\bar{x}_{k}
\end{aligned}
$$

Then

$$
\begin{aligned}
& p\left(\tilde{x}_{k}\right)=\Phi_{M_{k}}\left(x_{k}-\bar{x}_{k}\right) \\
& z_{k}=T_{k} H_{k} \tilde{x}_{k}+u_{k}
\end{aligned}
$$

where

$$
u_{k}=T_{k} v_{k}
$$

Expanding the error-covariance we have

$$
\begin{aligned}
& \left.E\left[x_{k}-\hat{x}_{k}\right)\left(x_{k}-\hat{x}_{k}\right)^{T}\right]=E\left[\tilde{x}_{k} \tilde{x}_{k}^{T}\right]-E\left[\left(\hat{x}_{k}-\bar{x}_{k}\right) \tilde{x}_{k}^{T}\right] \\
& -E\left[\tilde{x}_{k}\left(\hat{x}_{k}-\bar{x}_{k}\right)^{T}\right]+E\left[\left(\hat{x}_{k}-\bar{x}_{k}\right)\left(\hat{x}_{k}-\bar{x}_{k}\right)^{T}\right]
\end{aligned}
$$

The first term of (A6) is equal to $M_{k}$. From (13) the second term is written as

$$
\begin{aligned}
E\left[\left(\hat{x}_{k}-\bar{x}_{k}\right) \tilde{x}_{k}^{T}\right]= & \int_{R^{n}} \int_{R^{r}} H_{k}^{T} T_{k}^{T} A_{k} \Psi_{k}\left(z_{k}\right) \tilde{x}_{k}^{T} \\
& \cdot p\left(z_{k}, \tilde{x}_{k}\right) d \tilde{x}_{k} d z_{k}
\end{aligned}
$$

where $p\left(z_{k}, \tilde{x}_{k}\right)$ is a joint probability density function. Using the Bayes' rule

$$
p\left(z_{k}, \tilde{x}_{k}\right)=p\left(\tilde{x}_{k}\right) p_{u_{k}}\left(z_{k}-T_{k} H_{k} \tilde{x}_{k}\right)
$$

and the following relation

$$
\tilde{x}_{k} \Phi_{M_{k}}\left(\tilde{x}_{k}\right)=-M_{k}\left(\partial \Phi_{M_{k}}\left(\tilde{x}_{k}\right) / \partial \tilde{x}_{k}\right)
$$
and integrating by part, (A7) can be written as

$$
\begin{aligned}
E\left[\left(\hat{x}_{k}-\bar{x}_{k}\right) \tilde{x}_{k}^{T}\right]= & H_{k}^{T} T_{k}^{T} A_{k}\left[\int_{R^{r}}\left(\partial \Psi_{k}\left(z_{k}\right) / \partial z_{k}\right)^{T}\right. \\
& \left.\cdot p\left(z_{k}\right) d z_{k}\right] T_{k} H_{k} M_{k} \quad(\mathrm{~A} 10)
\end{aligned}
$$

$\left(\partial \Psi_{k}\left(z_{k}\right) / \partial z_{k}\right)^{T}$ is a diagonal matrix with the $i i$-th element equal to $\phi_{k}^{i}\left(z_{k}^{i}\right)^{\prime}$. Considering the definition of $\Omega_{k}$ we have

$$
E\left[\left(\hat{x}_{k}-\bar{x}_{k}\right) \tilde{x}_{k}^{T}\right]=H_{k}^{T} T_{k}^{T} A_{k} \Omega_{k} T_{k} H_{k} M_{k}
$$

By the same procedure we have

$$
E\left[\tilde{x}_{k}\left(\hat{x}_{k}-\bar{x}_{k}\right)^{T}\right]=M_{k} H_{k}^{T} T_{k}^{T} \Omega_{k} A_{k} T_{k} H_{k} \text { (A12) }
$$

The last term of (A6) is obtained from (13).

$$
\begin{aligned}
& E\left[\left(\hat{x}_{k}-\bar{x}_{k}\right)\left(\hat{x}_{k}-\bar{x}_{k}\right)^{T}\right]=H_{k}^{T} T_{k}^{T} A_{k} \\
& \quad \cdot\left[\int_{R^{r}} \Psi_{k}\left(z_{k}\right) \Psi_{k}\left(z_{k}\right)^{T} p\left(z_{k}\right) d z_{k}\right] A_{k} T_{k} H_{k}
\end{aligned}
$$

where $\Psi_{k}\left(z_{k}\right) \Psi_{k}\left(z_{k}\right)^{T}$ is a diagonal matrix with the $i i$-th element $\left(\psi_{k}^{i}\left(z_{k}^{i}\right)\right)^{2}$. By the definition of $\Lambda_{k}$, (A13) is equal to $H_{k}^{T} T_{k}^{T} A_{k} \Lambda_{k} A_{k} T_{k} H_{k} \quad$ Q. E. D.

\section{Appendix B: The proof of Theorem 1}

$E\left[e_{k}^{2}\right], \quad E\left[e_{k}^{2} I_{k+1}^{2}\right]$, and $E\left[e_{k}\left(I_{k+1}^{3}-I_{k+1}^{4}\right)\right]$ are expanded, respectively, as follows.

$$
\begin{aligned}
& E\left[e_{k}^{2}\right]=E\left[\xi_{k}\right]-2\left(K_{k} H-1\right) E\left[\eta_{k}\right]+F^{2} E\left[e_{k-1}^{2} I_{k}^{2}\right] \\
& \quad-2 F K_{k} b_{k} E\left[e_{k-1}\left(I_{k}^{3}-I_{k}^{4}\right)\right]+K_{k}^{2} b_{k} E\left[I_{k}^{2}\right] \\
& \quad+2 F E\left[e_{k-1} w_{k-1} I_{k}^{2}\right]-2 K_{k} b_{k} E\left[w_{k-1}\left(I_{k}^{3}-I_{k}^{4}\right)\right] \\
& \quad+\left(K_{k}^{2} H^{2}-2 K_{k} H\right) E\left[w_{k-1}^{2} I_{k}^{1}\right] \quad(\mathrm{B} 1) \\
& E\left[e_{k}^{2} I_{k+1}^{2}\right]=F^{2} E\left[e_{k-1}^{2} I_{k}^{2}\right]-2 F K_{k} b_{k} E\left[e_{k-1}\left(I_{k}^{3}-I_{k}^{4}\right)\right] \\
& \quad+K_{k}^{2} b_{k}^{2} E\left[I_{k}^{2}\right]+2 F E\left[e_{k-1} w_{k-1}^{2} I_{k}^{2}\right] \\
& \quad-2 K_{k} b_{k} E\left[w_{k-1}\left(I_{k}^{3}-I_{k}^{4}\right)\right]+E\left[w_{k-1}^{2}\right] \\
& \quad-E\left[w_{k-1}^{2} I_{k}^{1}\right]+E\left[e_{k}^{2}\left(I_{k+1}^{2}-I_{k}^{2}\right)\right] \\
& E\left[e_{k}\left(I_{k+1}^{3}-I_{k+1}^{4}\right)\right]=F E\left[e_{k-1}\left(I_{k}^{3}-I_{k}^{4}\right)\right]-K_{k} b_{k} E\left[I_{k}^{2}\right] \\
& \quad+E\left[w_{k-1}\left(I_{k}^{3}-I_{k}^{4}\right)\right]+E\left[\zeta_{k}\right] \quad(\mathrm{B} 3)
\end{aligned}
$$

If $E\left[e_{k-1}^{2}\right] \leqq \rho_{k-1}^{1}$, then from (21) and (29)

$$
\begin{aligned}
& L_{d}^{2}\left(\rho_{k-1}^{1}\right) \leqq E\left[\xi_{k}\right] \leqq U_{i}^{2}\left(\rho_{k-1}^{1}\right) \\
& L_{d}^{3}\left(\rho_{k-1}^{1}\right) \leqq E\left[\eta_{k}\right] \leqq U_{i}^{3}\left(\rho_{k-1}^{1}\right) \\
& L_{d}^{7}\left(\rho_{k-1}^{1}\right) \leqq E\left[I_{k}^{2}\right] \leqq U_{i}^{7}\left(\rho_{k-1}^{1}\right) \\
& L_{d}^{4}\left(\rho_{k-1}^{1}\right) \leqq E\left[e_{k-1} w_{k-1} I_{k}^{2}\right] \leqq U_{i}^{4}\left(\rho_{k-1}^{1}\right) \\
& L_{d}^{5}\left(\rho_{k-1}^{1}\right) \leqq E\left[w_{k-1}\left(I_{k}^{3}-I_{k}^{4}\right)\right] \leqq U_{i}^{5}\left(\rho_{k-1}^{1}\right) \\
& L_{d}^{6}\left(\rho_{k-1}^{1}\right) \leqq E\left[w_{k-1}^{2} I_{k}^{1}\right] \leqq U_{i}^{6}\left(\rho_{k-1}^{1}\right)
\end{aligned}
$$

and if $E\left[J_{k}\left(e_{k-1}\right)\right] \geqq \rho_{k-1}^{4}$, then from (30) and (31)

$$
\begin{aligned}
& E\left[J_{k+1}\left(\mathrm{e}_{k}\right)\right] \geqq L_{i}^{10}\left(\rho_{k-1}^{4}\right) \\
& L_{i}^{8}\left(\rho_{k-1}^{4}\right) \leqq E\left[e_{k}^{2}\left(I_{k+1}^{2}-I_{k}^{2}\right)\right] \leqq U_{d}^{8}\left(\rho_{k-1}^{4}\right) \\
& L_{i}^{9}\left(\rho_{k-1}^{4}\right) \leqq E\left[\zeta_{k}\right] \leqq U_{d}^{9}\left(\rho_{k-1}^{4}\right)
\end{aligned}
$$

Equations (42)-(45) are derived by using (B1)(B12), and (46) is obtained directly from (30) and (31).

Q. E. D: 